\title{
Transparencia en los municipios españoles: determinantes de la divulgación de información
}

\author{
Transparency in Spanish municipalities: \\ determinants of information disclosure \\ Francisca Tejedo-Romero / Francisca.Tejedo@uclm.es \\ https://orcid.org/0000-0002-2600-9826 \\ Universidad de Castilla-La Mancha, España \\ Joaquim Filipe Ferraz Esteves Araujo / jfilipe@eeg.uminho.pt \\ https://orcid.org/0000-0001-8531-6036 \\ Universidade do Minho, Portugal
}

\begin{abstract}
The changes occurred in the management systems of public administration at present allow greater accountability and transparency. The purpose of this paper is twofold; firstly, study the level of transparency in the Spanish Public Administration, in particular we will focus on local governments; and, secondly, to determine those factors or variables that are affecting the levels of transparency. The methodology used to analyze the level of transparency used the transparency index of municipalities build by Transparency International in Spain as a proxy. For the treatment of the data, univariate and multivariate analyses are performed. The pressures exerted by citizens and other stakeholders are leading municipalities to become more transparent in the process of creating legitimacy before citizens and stakeholders. Those municipalities, where citizens have greater access to the Internet, have more population, fiscal pressure is heavier and are governed by left-wing parties, are more pressured to be transparent.
\end{abstract}

Key words: information disclosure, transparency, access to information, municipalities, open government.

Resumen: Los cambios que han ocurrido en los sistemas de gestión de la Administración Pública están permitiendo una mejor divulgación de información y transparencia. El propósito de este trabajo es estudiar el nivel de transparencia en los municipios españoles y determinar aquellos factores o variables que están afectando dicho nivel. La metodología empleada utiliza como proxy el índice de transparencia (IT) de los ayuntamientos, elaborado por la organización Transparencia Internacional en España. Para el tratamiento de los datos, se lleva a cabo un análisis univariante y multivariante. Las presiones ejercidas por los ciudadanos y otros stakeholders están llevando a los municipios a ser cada vez más transparentes, para crear legitimidad ante ellos. Aquellos municipios donde los habitantes cuentan con un mayor acceso a Internet, tienen más populación, la presión fiscal es más elevada y una ideología más progresista, se encuentran con una mayor presión para ser transparentes.

Palabras clave: divulgación de información, transparencia, acceso a la información, municipios, Gobierno Abierto. 


\section{Introducción $^{1}$}

Las ideas de la Nueva Gestión Pública (NGP) han cambiado la cultura tradicional de la Administración Pública, abriéndose las organizaciones e incrementando los mecanismos de rendición de cuentas y transparencia informativa. Dicha transparencia es un mecanismo para mejorar el buen gobierno en los servicios públicos (Piotrowsky, 2007; Albalate, 2013; Bauhr y Grimes, 2014) y para aumentar la capacidad del público de tener acceso a la información relativa al gobierno (Roberts, 2006; Piotrowski y Van Ryzin, 2007). Se trata de decisiones del gobierno, encaminadas a incrementar la eficacia de la gestión, procurando aumentar los valores éticos de estos (Cooper y Yoder, 2002).

La transparencia permite una mejor comprensión de las políticas públicas, incrementa la confianza del público y reduce la corrupción (Park y Blenkinsopp, 2011). Los dirigentes y gestores públicos deben rendir cuentas de sus actividades y resultados, basándose en la accesibilidad y la divulgación de información (Albalate, 2013). La transparencia en la Administración Pública se ha incrementado en todo el mundo desde 1990 (WBG, 2007; USAID, 2011). Numerosas organizaciones internacionales han recomendado que los gobiernos deberían adoptar prácticas socialmente responsables y proporcionar información acerca de sus actividades (OCDE, 2006; UE, 2011). Recién en España se ha aprobado la Ley 19/2013, del 9 de diciembre, de Transparencia, Acceso a la Información Pública y Buen Gobierno,

que tiene por objeto ampliar y reforzar la transparencia de la actividad pública, regular y garantizar el derecho de acceso a la información relativa a aquella actividad y establecer las obligaciones de buen gobierno que deben cumplir los responsables públicos. Esta ley se aplica a todas las Administraciones públicas y a todo el sector público estatal, así como a otras instituciones, como son la Casa de Su Majestad el Rey, el Consejo General del Poder Judicial, el Tribunal Constitucional, el Congreso de los Diputados, el Senado, el Banco de España, el Defensor del Pueblo, el Tribunal de Cuentas, entre otros (Ley 19/2013, de 9 de diciembre, art. 1 y 2).

La Ley prevé una entrada en vigor escalonada, atendiendo a las especiales circunstancias que conlleva la aplicación de las diversas disposiciones (al día siguiente de la publicación de la misma, al año o a los dos años). En concreto, para las entidades locales disponían de un plazo máximo de dos años para adaptarse a las obligaciones contenidas en esta Ley.

1 Estudio llevado a cabo en el Centro de Investigación en Ciencias Políticas (UID / CPO / 00758/2013), Universidad de Minho y apoyado por la Fundación para la Ciencia y Tecnología y el Ministerio de Educación y Ciencia portuguesa a través de fondos nacionales. 
En cuanto a la transparencia en el ámbito local, es relevante para tener una mejor comprensión sobre el gobierno local (Piotrowski y Bertelli, 2010; Greco et al., 2012). El uso eficiente de los recursos públicos exige mecanismos más eficaces de transparencia en la toma de decisiones de los gobiernos locales. Esto está motivado por la presión de parte de los ciudadanos con la finalidad de reducir la corrupción y los abusos de poder.

El impacto de la crisis ha forzado a los municipios españoles a reducir la deuda pública. Además, hay restricciones financieras que exigen a los gobiernos locales un mayor control legal del déficit público (Pérez-López et al., 2013). En este sentido, los municipios deben aumentar la eficacia en la gestión de los recursos públicos y ser más transparentes.

No obstante, hay determinados factores políticos y económicos importantes para explicar el nivel de transparencia en el gobierno local (Alt et al., 2006; Piotrowski y Van Ryzin, 2007; Guillamón et al., 2011; Albalate; 2013; Navarro et al., 2014; Alcaraz-Quiles et al., 2015; Nevado-Gil y Gallardo-Vázquez, 2016). En este trabajo se analiza el nivel de transparencia e identifica aquellas características de los municipios que la están afectando. Nosotros elegimos llevar a cabo nuestro análisis en los municipios, porque los problemas con la transparencia son más frecuentes en este ámbito (Guillamón et al., 2011; Cuadrado-Ballesteros, 2014).

Con base en la teoría de la agencia y de la legitimidad, la presente investigación contribuye a la literatura sobre este tema, tratando de explicar los factores determinantes del nivel de transparencia en los municipios españoles. El objetivo es responder a las siguientes preguntas: a) ¿se ha incrementado el nivel de transparencia en los municipios españoles? y b) ¿qué factores influyen en el nivel de transparencia de dichos municipios?

Para alcanzar el objetivo se realizó un análisis descriptivo univariante y bivariante, y un análisis de regresión lineal multivariante. Se seleccionó un total de cien municipios que se incluyeron por primera vez en el IT municipal, elaborado por el organismo Transparencia Internacional-España, en concreto para el año 2008, y se han considerado los mismos ayuntamientos para obtener los datos correspondientes al año 2014.

Finalmente, tenemos un panel de datos con 200 observaciones. Los resultados indican que ha habido un incremento, por parte de los municipios de la muestra, en sus niveles de transparencia y que factores como el tamaño del municipio, la presión fiscal, el nivel de acceso a Internet de los pobladores, la ideología política del municipio y el efecto de la ideología del partido político de la región (comunidad autónoma) tienen un impacto significativo en el nivel de transparencia municipal. 
El resto del trabajo se organiza así: en la siguiente sección se revisa la literatura sobre la transparencia, su definición, los factores que la afectan, y se desarrollan las hipótesis. En la tercera sección se presenta la metodología de la investigación; en la cuarta, la descripción de los datos y el análisis empírico; y en la quinta, las principales conclusiones.

\section{Antecedentes teóricos y desarrollo de hipótesis}

El sector público español se divide en tres niveles: el Gobierno Central, 17 Gobiernos Regionales y 8.112 Gobiernos Locales, agrupados en 50 provincias. Los municipios son el nivel más bajo de la organización territorial del Estado, tienen personalidad jurídica y plena capacidad para el cumplimiento de sus fines. El gobierno y la administración de los municipios corresponde a sus respectivos ayuntamientos, integrados por alcaldes y concejales.

Para analizar la transparencia del Gobierno Local nos centramos en los municipios. En España, la reforma, iniciada por la Ley 57/2003 de Medidas para la Modernización del Gobierno Local, subraya la necesidad de impulsar la utilización de Tecnologías de la Información y la Comunicación para facilitar la participación y la comunicación con los vecinos (Serrano-Cinca et al., 2009; Guillamón et al. 2011; García et al., 2013); contribuyendo así con la transparencia informativa.

La literatura señala cuatro principios de transparencia: 1) la información sobre las actividades de las organizaciones públicas que afectan los intereses de los ciudadanos debe ser valiosa y estar a disposición del público; 2) la cantidad de información disponible deberá ser adecuada al grado en que esas organizaciones amenazan los intereses de los ciudadanos; 3 ) la información debe ser accesible para quienes la ocupan; y 4) las estructuras sociales, políticas y económicas de la sociedad deben organizarse de manera que permitan a la ciudadanía actuar (Birkinshaw, 2006; Ball, 2009; Fung, 2013).

La transparencia es un medio para mejorar la eficacia de las políticas públicas y la eficacia administrativa y de gestión (Hirsch y Osborne, 2000), conduciendo los asuntos públicos de forma abierta para el escrutinio público (Birkinshaw, 2006). Va más allá del mero acceso a la información, exigiendo que esta resulte comprensible para los interesados externos (Hood, 2006; Nevado-Gil y Gallardo-Vázquez, 2016).

A los efectos de este estudio, la transparencia es la capacidad del público para tener acceso a la información gubernamental (Roberts, 2006; Piotrowski y Van Ryzin, 2007; Piotrowski y Bertelli, 2010). Para explorar el nivel de transparencia pública en los gobiernos locales, vamos a utilizar el IT de los 
ayuntamientos españoles, publicado por Transparencia Internacional España (2018), para probar nuestras hipótesis.

La primera edición del IT se llevó a cabo en 2008 y evaluó los 100 municipios más grandes de España mediante un conjunto de 80 indicadores, agrupados en cinco áreas de transparencia: "información sobre las corporaciones municipales, relaciones con los ciudadanos y la sociedad, transparencia económico-financiera, transparencia en las contrataciones y servicios, y transparencia en materias de urbanismo y obras públicas" (Transparencia Internacional España, 2018). A partir de 2009 se analizan 110 municipios, y, desde 2012 se incorpora una nueva área de estudio relacionada con los indicadores de la ley de transparencia (en total 6 áreas).

Muchas investigaciones se han basado en la teoría de la agencia y de la legitimidad para explicar los motivos de la transparencia informativa (Zimmerman, 1977; Eisenhardt, 1989; Patten, 1992; Suchman, 1995; Shapiro, 2005; Tejedo-Romero, 2014).

En el marco de la teoría de la agencia, nosotros encontramos una relación de agencia en la Administración Pública, donde los agentes (representantes electos) deben actuar a favor del interés del principal (ciudadanos) (Lane, 2005). En esta relación surgen algunos problemas entre las partes cuyo origen está en la existencia de asimetrías de información (Eisenhardt, 1989). Los representantes electos tienen intereses diferentes a los de los ciudadanos y deben ser responsables de sus acciones ante estos, para demostrar que han actuado para el bien ciudadano, de acuerdo con sus responsabilidades (Lane, 2005; Shapiro, 2005).

Cuando esto no sucede, surge un conflicto de intereses entre las partes, generando los denominados "costes de agencia”. Una manera de reducir estos es mediante la revelación de información. La transparencia reduce las asimetrías de la información y aumenta el grado de confianza entre las partes; así, la confianza pública en los actores políticos limita los conflictos de agencia (Laswad et al., 2005). Por otro lado, el incremento del nivel de transparencia en la toma de decisiones y actividades gubernamentales aumenta la probabilidad de una mayor participación ciudadana y la democracia.

La teoría de la legitimidad es otra perspectiva para explicar la divulgación de información en la transparencia (Deegan, 2002; Serrano-Cinca et al., 2009; Rodríguez-Bolívar et al., 2013; Tejedo-Romero, 2014). Revelar información sobre las actividades de la organización es una forma de legitimar sus acciones ante los grupos de interés (stakeholders) (Archel et al., 2009) y también otorgar legitimidad a las organizaciones (Patten, 1992). De acuerdo con esta teoría, si la legitimidad de la organización se ve amenazada, esta 
divulgará información para promover su legitimidad ante los stakeholders (Deegan, 2006; Tejedo-Romero, 2014).

La revelación de información puede ser utilizada para recuperar la confianza de los ciudadanos y para cambiar la percepción que estos tienen acerca de la organización. Además, ofrece una imagen de buen gobierno en respuesta a las presiones generales, siendo una manera de asegurar la legitimidad y mejorar la reputación de los municipios (Pina et al., 2010).

Divulgar información por parte de los Gobiernos Locales ha sido una preocupación para los investigadores desde la década de 1970, en particular lo relativo a los factores que promueven una mayor y mejor difusión de información económico-financiera pública (Laswad et al., 2005; Alt et al., 2006; Cárcaba y García-García, 2010; Rodríguez-Bolívar et al., 2013; Nevado-Gil y Gallardo-Vázquez, 2016). A continuación, haremos referencia a una serie de factores que pueden influir en la divulgación de información, y, por tanto, en el nivel de transparencia de los ayuntamientos.

\section{Tecnologías de la Información}

En el marco de la teoría de la agencia, las Tecnologías de la Información, en concreto Internet, constituyen un medio que facilita la comunicación y la participación entre las partes (Caba-Pérez et al., 2008). Entre mayor acceso tengan los ciudadanos a Internet, es más probable que consulten la página web de su ayuntamiento (Gandía y Archidona, 2008; Pina et al., 2010; Alcaraz-Quiles et al., 2015).

En este sentido, los ayuntamientos se verían más presionados por los ciudadanos a ser más transparentes y a difundir más información con la finalidad de legitimar ante ellos sus acciones. Además, supondría menores asimetrías de información entre los ciudadanos y los ayuntamientos, reduciendo el posible conflicto de intereses que podría surgir entre ambos. Por lo tanto, proponemos la siguiente hipótesis:

H1: Existe una relación positiva entre el nivel de acceso a Internet de la ciudadania y el nivel de transparencia de los ayuntamientos

\section{Tamaño}

Un mayor tamaño del municipio implicaría más usuarios (ciudadanos) de los servicios públicos (Alcaraz-Quiles et al., 2015) y gestionar una mayor cantidad de fondos públicos. De acuerdo con la teoría de la agencia, se espera que 
los ayuntamientos estén interesados en revelar más información que permita a los ciudadanos y a otros grupos supervisar las acciones realizadas por sus representantes electos (Laswad et al., 2005; Benito et al., 2010; González et al., 2011; Nevado-Gil y Gallardo-Vázquez, 2016). Por otra parte, una mayor revelación de información implicará una mayor legitimidad del ayuntamiento ante los ciudadanos, quienes desean conocer en todo momento si sus recursos se han gestionado de manera eficaz y eficiente (Styles y Tennyson, 2007). Nuestra segunda hipótesis a contrastar es:

H2: Existe una relación positiva entre el tamaño del municipio y el nivel de transparencia de los ayuntamientos

Presión fiscal

De acuerdo con la teoría de la agencia, existe una relación positiva entre altos niveles de impuestos en los municipios y el nivel de transparencia fiscal de los ayuntamientos (Lassen, 2000). Los ciudadanos quieren obtener los mejores servicios públicos y pagar la menor cantidad de impuestos posible (Alcaraz-Quiles et al., 2015). Para reducir las asimetrías de información y aumentar el grado de confianza, los ayuntamientos revelarán más información con la finalidad de justificar a los ciudadanos que esa presión fiscal ha podido estar motivada por una mejora de los servicios públicos (Guillamón et al., 2011).

Por otro lado, altos niveles de transparencia y rendición de cuentas es un requisito para que los ayuntamientos demuestren la buena gestión de los recursos financieros, y los contribuyentes legitimen sus actuaciones. En este sentido, planteamos la siguiente hipótesis:

H3: Existe una relación positiva entre la presión fiscal del municipio y el nivel de transparencia de los ayuntamientos.

\section{Rivalidad politica}

La rivalidad política puede ser un determinante para que el partido que está gobernando en ese momento en el ayuntamiento sea más transparente. En aquellos municipios donde existen más partidos políticos, los grupos políticos de la oposición van a ejercer una mayor presión al grupo que gobierna (García-Sánchez et al., 2013; Nevado-Gil y Gallardo-Vázquez, 2016). Por tanto, el partido que gobierna, para legitimar sus actuaciones ante los 
grupos de la oposición, tenderán a ser más transparentes. Así, se reduce el posible conflicto de intereses que puede existir entre ambos. Y formulamos la siguiente hipótesis:

H4: Existe una relación positiva entre el nivel de rivalidad politica del ayuntamiento y su nivel de transparencia.

\section{Ideología politica}

Algunos investigadores señalan la existencia de una fuerte relación entre la ideología política y la transparencia (Albalate, 2013; García-Sánchez et al., 2013); no obstante, las investigaciones no han sido concluyentes. Por su parte, Guillamón et al. (2011) y García-Sánchez et al. (2013) indican que los partidos políticos de izquierda son más transparentes que los de derecha; en tanto, autores como Piotrowski y Van Ryzin (2007) y Vila i Vila (2013) advierten de que esta relación es ambigua, porque tanto los partidos progresistas como los conservadores están interesados en ser transparentes. Así, se establece la siguiente hipótesis:

H5: Existe una relación entre la ideología politica del partido que gobierna en el municipio y el nivel de transparencia municipal.

\section{Metodología de investigación}

En esta sección se presenta la muestra utilizada y se justifican las variables seleccionadas para probar las hipótesis del estudio empírico.

\section{Muestra}

La muestra para probar nuestras hipótesis comprende cien ayuntamientos, lo cuales fueron incluidos en 2008 en el primer IT publicado por Transparencia Internacional España (2018). Estos mismos ayuntamientos los hemos mantenido para el año 2014 y hemos obtenido un panel de datos balanceado de 200 observaciones $(\mathrm{N}=2 \times \mathrm{T}=2)$. 


\section{Las variables y la recolección de datos}

Variable dependiente: para explorar el nivel de transparencia pública de los gobiernos locales, la variable dependiente fue el IT, de los municipios españoles, publicado por Transparencia Internacional España (2018). El índice toma valores de entre 0 y 100 . Para efectos de análisis se ocupa el IT en una escala de 0 a 1 . La información para crear la variable dependiente se tomó de la página web de Transparencia Internacional España (2018).

Variables independientes: sobre la base de estudios previos de los factores determinantes que pueden influir en la transparencia, se seleccionaron las siguientes variables que podrían contribuir a aumentar la divulgación de información en los municipios. Los datos fueron tomados del Instituto Nacional de Estadística (2014) y del Ministerio de Economía y Administraciones Públicas de España (2014).

- Tecnologías de la Información: variable numérica que refleja el número de personas con acceso a Internet, dividido por el total de habitantes del municipio.

- Tamaño: variable numérica que refleja el número de habitantes del municipio.

- Presión fiscal: variable numérica que representa el total de impuestos directos e indirectos de los ayuntamientos per cápita.

- Rivalidad política: variable numérica que representa el número de partidos políticos que se presentaron a las elecciones municipales. Para el año 2008 se tomaron los datos relativos a las elecciones locales de 2007, y para los del año 2014 se obtuvieron los datos correspondientes a los comicios locales de 2011.

- Ideología política: variable dummy que toma el valor de 1 en caso de que el partido que gobierna en el ayuntamiento sea progresista y 0 en caso de ser conservador. Para el año 2008 se tomaron los datos relativos a las elecciones locales de 2007 y, para los del año 2014 se obtuvieron los datos correspondientes a los comicios locales de 2011.

Variables de control: hemos incorporado el efecto del partido regional para saber si la transparencia está influida por la ideología política del partido que gobierna en la región (en la Comunidad Autónoma). Esta variable toma el valor 1 cuando coincide la ideología política del ayuntamiento y de la región, y 0 en caso contrario. Y el año, para conocer si los niveles de transparencia han sido más significativos en 2008 o en 2014. 


\section{Modelo de investigación}

El principal objetivo de este trabajo es analizar los factores que afectan el nivel de transparencia en los años 2008 y 2014, por lo que se adoptó el método multivariante de regresión lineal. Esto se prueba empíricamente utilizando el siguiente modelo econométrico:

Indice de Transparencia ${ }_{i t}=B_{0}+\beta_{1}$ Tecnologias de Información ${ }_{i t}+\beta_{2}$ Tamaño $\mathrm{t}_{i}+\beta_{3}$ Presión Fiscal ${ }_{i t}+\beta_{4}$ Rivalidad Politica $_{i t}+\beta_{5}$ Ideología $^{i l}$ Politica $_{i t}+\beta_{6}$ Partido Regional $_{i t}+\beta$ Año ${ }_{i t}+\varepsilon_{i}$ dónde:

$i$ representa el ayuntamiento, $t$ es el año, $\beta$ representan los parámetros a estimar y $\varepsilon$ es el término de error.

Los datos fueron procesados con el software econométrico STATA 19.1.

\section{Resultados y discusión}

Esta sección proporciona resultados empíricos de nuestro estudio mediante el uso de tres análisis. En primer lugar, el análisis descriptivo presenta las principales características de los municipios españoles incluidos en la muestra. A continuación, haremos un análisis bivariante para mostrar la correlación bivariada entre las variables dependientes e independientes. Por último, haremos un análisis multivariado para tener evidencia sobre la relación entre las variables propuestas en el modelo econométrico.

\section{Análisis descriptivo}

En la Tabla $1^{2}$ se presentan los principales estadísticos descriptivos (media, desviación típica, valor máximo y mínimo) para las variables dependiente, independientes y de control. Debido a la existencia de valores extremos, se han winsorizado la variable presión fiscal y tamaño de la población a un nivel del $10 \%$ y se han tomado sus logaritmos.

En el panel A de la Tabla 1 se expone un valor medio del IT para las 200 observaciones de 0.6868 . Un valor bastante significativo que podría estar motivado por el efecto de la implantación de la Ley de transparencia. El impacto que ha podido tener la entrada en vigor de dicha ley se constata en el valor medio que adquiere el IT en los dos años, pasando de un 0.5213 en 2008 a un 0.8523 en 2014 .

2 Todas las tablas se encuentran en el Anexo, al final del presente artículo (Nota del editor). 
Del análisis descriptivo, cabe señalar que también se ha incrementado el acceso a Internet en los hogares españoles, pasando de un valor medio de 77.4940 en 2008 a un 93.8000 en 2014; es decir, la mayoría de los hogares dispone de acceso a Internet. En relación con la presión fiscal de los ayuntamientos, los niveles de imposición directa e indirecta han sido muy similares para 2008 y 2014; esto puede deberse al periodo de crisis en el que hemos estado inmersos, pues a pesar de intentar reducir la deuda pública, la presión fiscal se ha mantenido prácticamente constante con el fin de no encarecer más la situación económica de los ciudadanos.

Respecto a las variables relacionadas con la política, se observa un incremento en el número medio de partidos políticos bastante considerable, pasando de una media de 9 partidos por municipio, a 11 partidos por municipio. La presión ejercida por el movimiento de "los indignados" en plena crisis quizá haya podido motivar dicho incremento.

En el panel B de la Tabla 1, se observa que, si bien en 2008 lideraban en un 54\% de los ayuntamientos partidos de ideología política progresista, en 2014 se produce una reducción prácticamente de la mitad, gobernando tan sólo un $25 \%$ con esta ideología. Tal vez la presión de nuevos movimientos políticos, a nivel local, ha provocado un cambio hacia ideologías más conservadoras. Además, para las 200 observaciones, se muestra que en un 58\% de los ayuntamientos la ideología que lidera es del mismo signo que la que gobierna a nivel regional (a nivel de la comunidad autónoma).

\section{Análisis bivariado}

La Tabla 2 presenta los coeficientes de correlación de Pearson entre las variables anteriores, y la última columna refleja los valores de los factores de inflación de varianza (FIV).

Se muestra en la Tabla 2 que las relaciones bivariantes son consistentes con el marco teórico desarrollado anteriormente y con los resultados obtenidos en estudios previos (Guillamón et al., 2011; Albalate, 2013; Caamaño et al., 2013; Nevado-Gil y Gallardo-Vázquez, 2016). Sin embargo, no fue posible confirmar una correlación significativa entre el índice de transparencia y la ideología política y el efecto regional.

Además, los valores no indican colinealidad. Un problema de colinealidad se considera grave si un coeficiente de correlación de pares es mayor que 0,80 (Gujarati, 1995: 335). Por otra parte, para examinar la presencia o ausencia de multicolinealidad, también se analizaron los factores de inflación de varianza (FIV). Los valores obtenidos indican ausencia de multicolinealidad; valores de la FIV por debajo de 5 son aceptables (Hair et al., 2006). 


\section{Análisis multivariante}

En la Tabla 3 se exponen los resultados del modelo pool de regresión lineal para datos de panel (también denominado modelo agrupado). En la tercera columna se reflejan los resultados para la muestra completa de las $200 \mathrm{ob}$ servaciones. En la columna 4 figuran los resultados, considerando exclusivamente el año 2008, y en la columna 5 se presentan los resultados para el año 2014, ambos con 100 observaciones cada uno (análisis de corte transversal).

Para la validación del modelo global, hemos realizado varios test: el de Breusch-Pagan / Cook-Weisberg para la heterocedasticidad, el de omisión de variables de Ramsey y la prueba de multicolinealidad (FIV, ya mencionada en la sección anterior). La significación global del modelo se prueba a través del estadístico $F$.

Así, la prueba de Breusch-Pagan/ Cook-Weisberg reveló ausencia de heterocedasticidad en los resultados $\left(\mathrm{X}_{(32)}^{2}=25.44\right.$; $\mathrm{p}$-valor $\left.=0.7879\right)$. Por otra parte, la prueba de especificación del modelo de Ramsey muestra que no se ha omitido ninguna variable Reset $\left(\mathrm{F}_{(3,189)}=0.42\right.$; $\mathrm{p}$-valor $=0.7410$. El estadístico $F\left(\mathrm{~F}_{(7,16)}=138.49\right.$; p-valor $\left.=0.0000\right)$ resulta significativo.

Como se refleja en la columna 3 de la Tabla 3 , los resultados reflejan que las Tecnologías de la Información tienen un impacto positivo y significativo en el nivel de transparencia de los municipios a un nivel del 10\% ( $\beta$ $\left.{ }_{1}=0.0040\right)$. Esto confirma nuestra Hipótesis 1 , ya que demuestra que los municipios donde los ciudadanos tienen un mayor acceso a las tecnologías de información, en concreto a Internet, son más transparentes. Esto es consistente con los hallazgos de Caba-Pérez et al. (2008), Gandía y Archidona (2008) y Alcaraz-Quiles et al. (2015).

Se observa que la variable tamaño (columna 3 de la Tabla 3 ) presenta un efecto positivo para un nivel de significancia del $1 \%\left(\beta_{2}=0.0845\right)$. Esto corrobora nuestra Hipótesis 2, al demostrar que los municipios de mayor tamaño son los que mayores niveles de transparencia presentan. Esto es consistente con los resultados obtenidos en otros trabajos (Benito et al., 2010; Alcaraz-Quiles et al., 2015; Nevado-Gil y Gallardo-Vázquez, 2016).

En relación con la variable presión fiscal (columna 3 de la Tabla 3 ) también ha resultado significativa para un nivel del $1 \%$ y tiene una relación positiva con el IT $\left(\beta_{3}=0.1558\right)$. Por lo tanto, aceptamos nuestra Hipótesis 3 , quedando testado que los municipios que ejercen mayor presión fiscal sobre los ciudadanos son los más transparentes.

Sin embargo, si observamos la columna 3 de la Tabla 3, no se encuentra ninguna relación significativa entre la rivalidad politica y el nivel de 
transparencia de los ayuntamientos, aunque tiene un efecto positivo $\left(\beta_{4}=\right.$ 0.0007). Así, rechazamos nuestra Hipótesis 4.

Por otro lado, hemos encontrado un efecto positivo y significativo para un nivel de aceptación del $1 \%$ respecto a nuestra variable ideología $\left(\beta_{5}=\right.$ 0.0630). Esto confirma nuestra Hipótesis 5, pues demuestra que la ideología política tiene relación con el nivel de transparencia de los ayuntamientos. En concreto, nuestros resultados confirman los hallazgos de Guillamón et al. (2011) y García-Sánchez et al. (2013), quienes hallaron que los ayuntamientos más progresistas son los que presentan mayores niveles de transparencia.

Finalmente, nuestras variables de control (columna 3 de la Tabla 3 ) reflejan que el partido regional tiene un efecto sobre el nivel de transparencia del ayuntamiento. Así, aquellos municipios, cuya ideología política es la misma que en la Comunidad Autónoma, son más transparentes $\left(\beta_{6}=0.401\right)$ para un nivel de significación del $10 \%$. Asimismo, los ayuntamientos son más transparentes en el año 2014 en relación con el 2008, para un nivel de significación del $1 \%\left(\beta_{7}=0.2728\right)$.

Adicionalmente a nuestro modelo global, hemos querido testar el modelo para cada uno de los años por separado. Así, para 2008 han resultado significativas las variables: tamaño, presión fiscal e ideología; y para 2014: Tecnologías de la Información, presión fiscal, ideología y el efecto del partido regional.

\section{Conclusiones}

Este trabajo pretende contribuir a la literatura relativa a la transparencia. Bajo los postulados de la teoría de la agencia y de la legitimidad, el principal objetivo fue realizar un análisis sobre el nivel de transparencia de los ayuntamientos españoles y las características que pueden incidir en dichos niveles de transparencia.

Se empleó un modelo de regresión agrupado (pool) con 200 observaciones correspondientes a datos de un panel balanceado de 100 municipios para los años 2008 y 2014. Nuestros resultados muestran que, debido a las asimetrías de información existentes entre los gobernantes y los ciudadanos, los municipios españoles están suministrando más información, son más transparentes en 2014 que en 2008. Las presiones ejercidas por los ciudadanos y otros stakeholders están llevando a los municipios a ser cada vez más transparente con las actividades que realizan, en vías de crear legitimidad ante aquellos. Esto deriva en una mayor confianza de los ciudadanos y otros grupos de interés en los ayuntamientos, una mejor reputación y un buen gobierno municipal con valores más éticos y responsables. 
Además, la entrada en vigor de la Ley de transparencia ha llevado a que los municipios se vayan adaptando a ella y vayan suministrando de manera voluntaria determinada información antes de su implantación obligatoria.

Hemos podido evidenciar que en los municipios donde sus habitantes tienen un mayor acceso a Internet, lo ayuntamientos son más transparentes. El tamaño del municipio también está relacionado con los niveles de transparencia de este, si bien los ayuntamientos, en este caso, se encuentran presionados por un mayor número de ciudadanos.

Por otro lado, aquellos municipios donde la presión fiscal es más elevada parecen estar más presionados para ser transparentes ante sus ciudadanos y evitar el posible conflicto de intereses entre ellos. Además, es una manera de que el ayuntamiento legitime ante los ciudadanos de que están actuando conforme a la ley.

También, hemos podido corroborar que los ayuntamientos gobernados por ideologías más progresistas son los que más información revelan y los más transparentes. Además, tiene un efecto positivo el hecho de que el gobierno regional tenga la misma ideología que el gobierno municipal.

Finalmente, el año de referencia de estudio tiene una incidencia significativa en los niveles de transparencia de los ayuntamientos.

\section{Referencias}

Albalate del Sol, Daniel (2013), "The institutional, economic and social determinants of local government transparency", en Journal of Economic Policy Reform, vol. 16 núm. 1, Reino Unido: Routledge. Disponible en: http://www.tandfonline.com/doi/abs/10.1 080/17487870.2012.759422. [1 de julio de 2017].

Alcaraz-Quiles, Francisco et al. (2015), "Factors determining online sustainability reporting by Local Governments", en International Review of Administrative Sciences, vol. 81 núm. 1, Estados Unidos: Sage. Disponible en: http://journals.sagepub.com/ doi/10.1177/0020852314541564 [ 1 de julio de 2017].

Alt, James et al. (2006), "The causes of fiscal transparency: evidence from the U.S. states", en IMF Staff Papers, vol. 53, Estados Unidos: International Monetary Fund.

Archel, Pablo et al. (2009), "Social Disclosure, Legitimacy Theory and the Role of the State", en Accounting, Auditing \& Accountability Journal, vol. 22 núm. 8, Reino Unido: Emerald Group Publishing. Disponible en: http://www.emeraldinsight.com/doi/ abs/10.1108/09513570910999319 [16 de julio de 2017].

Ball, Carolyn (2009), "What is transparency?", en Public Integrity, vol. 11, núm. 4, Reino Unido: Taylor \& Francis. Disponible en: http://www.tandfonline.com/doi/ abs/10.2753/PIN1099-9922110400 [16 de julio de 2017].

Bauhr, Monika y Grimes, Marcia (2014), "Indignation or resignation: the implications of transparency for societal accountability", en Governance, vol. 27 núm. 2, Reino Unido: Blackwell. Disponible en: http://onlinelibrary.wiley.com/doi/10.1111/gove.12033/ abstract [16 de julio de 2017]. 
Francisca Tejedo-Romero y Joaquim Filipe Ferraz Esteves Araujo. Transparencia en los municipios españoles: determinantes de la divulgación de información

Benito, Bernardino et al. (2010), "Explaining differences in efficiency: an application to Spanish municipalities", en Applied Economics, vol. 42, Reino Unido: Routledge. Disponibleen:http://www.tandfonline.com/doi/abs/10.1080/00036840701675560 [28 de julio de 2017].

Birkinshaw, Patrick (2006), "Freedom of Information and Openness: Fundamental Human Rights?", en Administrative Law Review, vol. 58, Estados Unidos: American Bar Association.

Caamaño-Alegre, José et al. (2013), "Budget transparency in local governments: an empirical analysis”, en Local Government Studies, vol. 9 núm. 2, Reino Unido: Frank Cass. Disponible en: http://www.tandfonline.com/doi/abs/10.1080/03003930.201 2.693075 [ 1 de julio de 2017].

Caba Pérez, Carmen et al. (2008), "e-Government process and incentives for online public financial information”, en Online Information Review, vol. 32 núm. 3, Reino Unido: Emerald Group Publishing. Disponible en: http://www.emeraldinsight.com/doi/abs $/ 10.1108 / 14684520810889682$ ?journalCode=oir [16 de julio de 2017].

Cárcaba-García, Ana y García-García, Jesús (2010), "Determinants of Online Reporting of Accounting Information by Spanish Local Government Authorities", en Local Government Studies, vol. 36, núm. 5, Reino Unido: Frank Cass Publishers. Disponible en: http://www.tandfonline.com/doi/abs/10.1080/03003930.2010.506980 [1 de julio de 2017].

Cooper, Terry y Yoder, Diane (2002), "Public management ethics standards in a transnational world”, en Public Integrity, vol. 4, núm. 4, Reino Unido: Taylor \& Francis. Disponible en: http://www.tandfonline.com/doi/abs/10.1080/15580989.2002.11770926 [28 de julio de 2017].

Cuadrado-Ballesteros, Beatriz (2014), "The impact of functional decentralization and externalization on local government transparency", en Government Information Quarterly, vol. 31, núm. 2, Reino Unido: Elsevier. DOI: https://doi.org/10.1016/j. giq.2013.10.012 [28 de julio de 2017].

Deegan, Craig (2002), "Introduction: the legitimising effect of social and environmental disclosure - a theoretical foundation", en Accounting, Auditing \& Accountability Journal, vol. 15, núm. 3, Reino Unido: Emerald Group Publishing.

Deegan, Craig (2006), Financial Accounting Theory, Australia: McGraw-Hill.

Eisenhardt, Kathleen (1989), "Agency Theory: An Assessment and Review”, en Academy of Management Review, vol. 14, núm. 1, Estados Unidos: Academy of Managament. Disponible en: http://www.jstor.org/stable/258191 [1 de julio de 2017].

UE (Unión Europea) (2011), A renewed EU strategy 2011-14 for Corporate Social Responsibility, Bélgica: Unión Europea.

Fung, Archon (2013), "Infotopia: Unleashing the Democratic Power of Transparency", en Politics \& Society, vol. 41, núm. 2, Estados Unidos: Sage. Disponible en: http:// journals.sagepub.com/doi/10.1177/0032329213483107 [16 de julio de 2017].

Gandía, Juan y Archidona, María (2008), "Determinants of web site information by Spanish city councils", en Online Information Review, vol. 32, núm. 1, Reino Unido: Emerald Group Publishing. Disponible en: http://www.emeraldinsight.com/doi/ abs/10.1108/14684520810865976 [1 de julio de 2017].

García-Sánchez, Isabel María et al. (2013), "Determinants of corporate social disclosure in Spanish local governments”, en Journal of Cleaner Production, vol. 39, Holanda: Elsevier. Disponible en: https://doi.org/10.1016/j.jclepro.2012.08.037 [28 de julio de 2017]. 
Convergencia Revista de Ciencias Sociales, núm. 78, 2018, Universidad Autónoma del Estado de México

González, Eduardo et al. (2011), "Measuring quality of life in Spanish municipalities”, en Local Government Studies, vol. 37, núm. 2, Reino Unido: Frank Cass. Disponible en: http:// www.tandfonline.com/doi/abs/10.1080/03003930.2011.554826 [1 de julio de 2017].

Greco, Giulio et al. (2012), "From Tuscany to Victoria: Some Determinants of Sustainability Reporting by Local Councils", en Local Government Studies, vol. 38 núm. 5, Reino Unido: Frank Cass. Disponible en: http://www.tandfonline.com/doi/abs/10.1080/0 3003930.2012.679932 [16 de julio de 2017].

Guillamón, María Dolores et al. (2011), “The determinants of local government's financial transparency", en Local Government Studies, vol. 37, núm. 4, Reino Unido: Frank Cass. Disponible en: http://www.tandfonline.com/doi/abs/10.1080/03003930.2011.588 704 [16 de julio de 2017].

Gujarati, Damodar (1995), Basic econometrics, Estados Unidos: McGraw Hill.

Hair, Joseph et al. (2006), Multivariate data analysis, vol. 6, Estados Unidos: Pearson Prentice Hall.

Hirsch, Werner y Osborne, Evan (2000), "Privatization of government services: Pressuregroup resistance and service transparency”, en Journal of Labor Research, vol. 21, núm. 2, Estados Unidos: Transaction Publishiers. DOI: https://doi.org/10.1007/s12122000-1050-z [28 de julio de 2017].

Hood, Christopher (2006), “Transparency in Historical Perspective”, en Hood, Christopher y Heald, David [eds.], Transparency: The Key to Better Governance?, Inglaterra: Oxford University Press.

Instituto Nacional de Estadística (2014), INEbase. Padrón municipal. Disponible en: http:// www.ine.es/jaxiT3/Tabla.htm? $\mathrm{t}=2855 \& \mathrm{~L}=0$ [8 de enero de 2018].

Lane, Jan-Erik (2005), Public administration and public management: the principal-agent perspective, Inglaterra: Taylor \& Francis.

Lassen, David Dreyer (2000), Political accountability and the size of government: Theory and cross-country evidence, Dinamarca: Economic Policy Research Unit, Institute of Economics, University of Copenhagen.

Laswad, Fawzi et al. (2005), "Determinants of voluntary Internet financial reporting by local government authorities", en Journal of Accounting and Public Policy, vol. 24, núm. 2, Holanda: Elsevier. DOI: https://doi.org/10.1016/j.jaccpubpol.2004.12.006 [28 de julio de 2017].

Ley 19/2013 (2013), de 9 de diciembre, de Transparencia, Acceso a la información pública y Buen Gobierno. BOE, núm. 295, 10 de diciembre. Disponible en: https://www.boe.es/ buscar/act.php?id=BOE-A-2013-12887 [15 de septiembre de 2017].

Ministerio de Economía y Administraciones Públicas de España (2014),Datossobrepresupuestos y sus liquidaciones de las entidades locales. Disponible en: http://serviciostelematicosext. minhap.gob.es/sgcal/entidadeslocales/ [25 de septiembre de 2017].

Navarro, Andrés et al. (2014), "Transparency of sustainability information in local governments: English-speaking and Nordic cross-country analysis", en Journal of Cleaner Production, vol. 64, Holanda, Elsevier. DOI: https://doi.org/10.1016/j. jclepro.2013.07.038 [28 de julio de 2017].

Nevado-Gil, María Teresa y Gallardo-Vázquez, Dolores (2016), “Información sobre Responsabilidad Social contenida en las páginas webs de los ayuntamientos. Estudio en la región del Alentejo", en Revista Española de Documentación Cientifica, vol. 39, núm. 4, España: Centro Nacional de Información y Documentación Científica. DOI: https://doi.org/10.3989/redc.2016.4.1353 [16 de julio de 2017]. 
OCDE (Organización para la Cooperación y el Desarrollo Económico) (2006), Intellectual Assets and Value Creation: Implications for Corporate Reporting, Francia: Corporate Affairs Division, Directorate for Financial and Enterprise Affairs.

Park, Heungsik y Blenkinsopp, John (2011), "The roles of transparency and trust in the relationship between corruption and citizen satisfaction", en International Review of Administrative Sciences, vol. 77, núm. 2, Estados Unidos: Sage. DOI: https://doi. org/10.1177/0020852311399230 [28 de julio de 2017].

Patten, Dennis (1992), "Intra-industry environmental disclosures in response to the Alaskan oil spill: a note on legitimacy theory", en Accounting, Organizations and Society, vol. 17, Reino Unido: Elsevier. DOI: https://doi.org/10.1016/0361-3682(92)90042-Q [16 de julio de 2017].

Pérez-López, Gemma et al. (2013), "Deuda viva municipal en un contexto de crisis económica: Análisis de los factores determinantes y de las formas de gestión”, en Revista de Contabilidad, vol. 16, núm. 2, Reino Unido: Elsevier. DOI: https://doi. org/10.1016/j.rcsar.2012.12.001 [28 de julio de 2017].

Pina, Vicente et al. (2010), "Is E-government Promoting Convergence Towards More Accountable Local Governments?", en International Public Management Journal, vol. 13, núm. 4, Reino Unido: Taylor \& Francis. Disponible en: http://www.tandfonline. com/doi/abs/10.1080/10967494.2010.524834 [16 de julio de 2017].

Piotrowski, Suzanne y Van Ryzin, Gregg (2007), "Citizen attitudes toward transparency in local government", en American Review of Public Administration, vol. 37, Estados Unidos: Sage. DOI: https://doi.org/10.1177/0275074006296777 [28 de julio de 2017].

Piotrowski, Suzanne (2007), Governmental Transparency in the Path of Administrative Reform, Estados Unidos: State University of New York Press.

Piotrowski, Suzanne y Bertelli, Anthony (2010), Measuring Municipal Transparency. Paper presentado en el 14th IRSPM Conference, 7 al 9 abril, Suiza.

Roberts, Alasdair (2006), Blacked out: Government secrecy in the information age, Estados Unidos: Cambridge University Press.

Rodríguez-Bolívar, Manuel Pedro et al. (2013), "Determinants of Financial Transparency in Government", en International Public Management Journal, vol. 16, núm. 4, Reino Unido: Taylor \& Francis. Disponible en: http://www.tandfonline.com/doi/abs/10.1 080/10967494.2013.849169 [16 de julio de 2017].

Serrano-Cinca, Carlos et al. (2009), "Factors influencing e-disclosure in local public administrations", en Environment and Planning C: Government and Policy, vol. 27, núm. 2, Reino Unido: Pion. DOI: https://doi.org/10.1068/c07116r [16 de julio de 2017].

Shapiro, Susan (2005), "Agency Theory", en Annual Review of Sociology, vol. 31, Estados Unidos: Annual Reviews. Disponible en: http://www.annualreviews.org/ doi/10.1146/annurev.soc.31.041304.122159 [28 de julio de 2017].

Styles, Alan y Tennyson, Mack (2007), “The Accessibility of Financial Reporting of U.S. Municipalities on the Internet", en Journal of Public Budgeting, Accounting and Financial Management, vol. 19, núm. 1, Estados Unidos: PrAcademics Press. Disponible en: http://www.emeraldinsight.com/doi/abs/10.1108/JPBAFM-19-012007-B003 [28 de julio de 2017].

Suchman, Mark (1995), "Managing legitimacy: strategic and institutional approaches", en Academy of Management Review, vol. 20, núm. 3, Estados Unidos: Academy of Management. Disponible en: http://www.jstor.org/stable/258788 [16 de julio de 2017]. 
Tejedo-Romero, Francisca (2014), "Información del conocimiento organizacional a través de los informes anuales publicados en las páginas web de las empresas", en Revista Española de Documentación Cientifica, vol. 37, núm. 1, España: Centro Nacional de Información y Documentación Científica. DOI: http://dx.doi.org/10.3989/ redc.2014.1.1068 [28 de diciembre de 2014].

Transparencia Internacional España (2018), "Metodología del Índice de Transparencia de los Ayuntamientos 2017”. Disponible en: https://transparencia.org.es/wp-content/ uploads/2017/02/metodologia_ita-2017.pdf [12 de enero de 2018].

USAID (United States Agency for International Development) (2011), Fundamentals of NGO Financial Sustainability, Estados Unidos: Abt Associates.

Vila i Vila, Jordi (2013), "Determinantes de la transparencia contable en los municipios", en Auditoría Pública, vol. 60, España: Cámara de Comptos de Navarra.

WBG (World Bank Group) (2007), Environmental, Health, and Safety (EHS) Guidelines, Estados Unidos: Environment and Social Development Department, International Finance Corporation.

Zimmerman, Jerold (1977), "The municipal accounting maze: An analysis of political incentives", en Journal of Accounting Research, vol. 15, Reino Unido: Blackwell Publishing. Disponible en: http://www.jstor.org/stable/2490636 [16 de julio de 2017]. 


\section{Anexo}

\section{Tabla 1}

\section{Análisis descriptivo de las variables}

\begin{tabular}{|c|c|c|c|c|c|}
\hline Variable & Observ. & Media & $\begin{array}{l}\text { Desviación } \\
\text { típica }\end{array}$ & Mínimo & Máximo \\
\hline \multicolumn{6}{|c|}{ Panel A. Variables continuas } \\
\hline \multicolumn{6}{|c|}{ Índice de Transparencia } \\
\hline 2008 y 2014 & 200 & 0.6868 & 0.2424 & 0.1250 & 1.0000 \\
\hline 2008 & 100 & 0.5213 & 0.1776 & 0.1250 & 0.9060 \\
\hline 2014 & 100 & 0.8523 & 0.1768 & 0.2880 & 1.0000 \\
\hline \multicolumn{6}{|c|}{ Tecnologías de la Información } \\
\hline 2008 y 2014 & 200 & 85.6470 & 9.3256 & 70.3000 & 96.9000 \\
\hline 2008 & 100 & 77.4940 & 6.0576 & 70.3000 & 85.8000 \\
\hline 2014 & 100 & 93.8000 & 1.9573 & 90.4000 & 96.9000 \\
\hline \multicolumn{6}{|l|}{ Tamaño } \\
\hline 2008 y 2014 & 200 & 11.8419 & 0.5095 & 11.2260 & 12.7131 \\
\hline 2008 & 100 & 11.8357 & 0.5142 & 11.2260 & 12.7131 \\
\hline 2014 & 100 & 11.8481 & 0.5073 & 11.2260 & 12.7131 \\
\hline \multicolumn{6}{|l|}{ Presión fiscal } \\
\hline 2008 y 2014 & 200 & 6.3512 & 0.1917 & 6.0568 & 6.6488 \\
\hline 2008 & 100 & 6.3131 & 0.1905 & 6.0568 & 6.6488 \\
\hline 2014 & 100 & 6.3894 & 0.1861 & 6.0568 & 6.6488 \\
\hline \multicolumn{6}{|c|}{ Rivalidad política } \\
\hline 2008 y 2014 & 200 & 9.8650 & 4.1946 & 3.0000 & 25.0000 \\
\hline 2008 & 100 & 8.9700 & 3.7076 & 3.0000 & 25.0000 \\
\hline 2014 & 100 & 10.7600 & 4.4724 & 4.0000 & 25.0000 \\
\hline \multicolumn{6}{|c|}{ Panel B. Variables dummies } \\
\hline & Observ. & 1 & 0 & & \\
\hline \multicolumn{6}{|l|}{ Ideología (\%) } \\
\hline 2008 y 2014 & 200 & 40 & 60 & & \\
\hline 2008 & 100 & 54 & 46 & & \\
\hline 2014 & 100 & 25 & 75 & & \\
\hline \multicolumn{6}{|c|}{ Efecto regional (\%) } \\
\hline 2008 y 2014 & 200 & 58 & 42 & & \\
\hline 2008 & 100 & 61 & 39 & & \\
\hline 2014 & 100 & 55 & 45 & & \\
\hline
\end{tabular}

Fuente: Elaboración propia. 


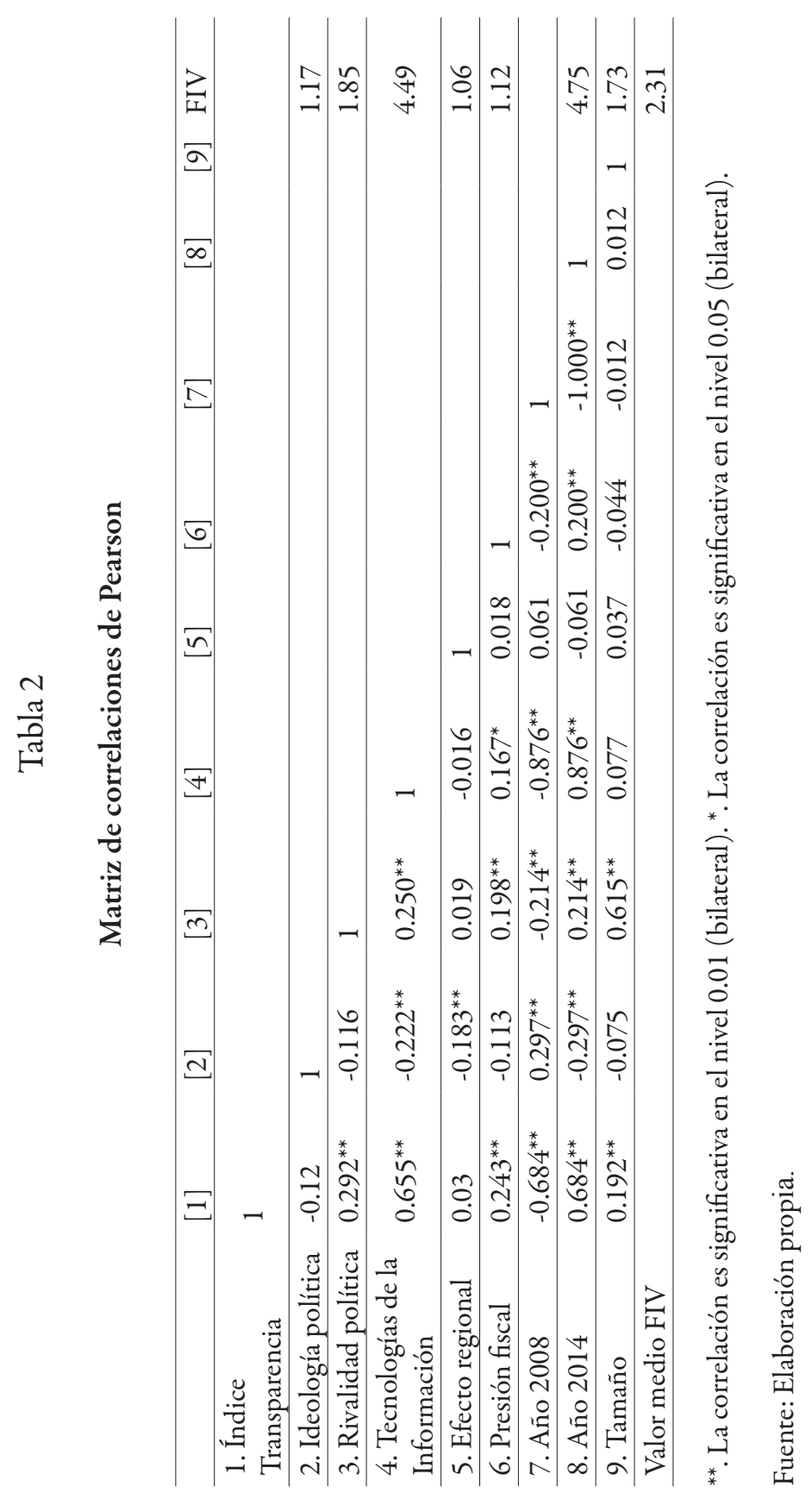




\section{Tabla 3}

\section{Modelo de regresión lineal (pool)}

\begin{tabular}{|c|c|c|c|c|}
\hline & \multicolumn{4}{|c|}{ VARIABLE DEPENDIENTE: Índice de Transparencia } \\
\hline $\begin{array}{l}\text { VARIABLES } \\
\text { INDEPENDIENTES }\end{array}$ & Predic. signo & $2008-2014$ & 2008 & 2014 \\
\hline \multirow{2}{*}{$\begin{array}{l}\text { Tecnologías de la Infor- } \\
\text { mación }\end{array}$} & \multirow{2}{*}{$\mathrm{H} 1(+)$} & $0.0040^{*}$ & 0.0035 & $0.0162^{*}$ \\
\hline & & $(0.0022)$ & $(0.0028)$ & $(0.0088)$ \\
\hline \multirow{2}{*}{ Tamaño } & \multirow{2}{*}{$\mathrm{H} 2(+)$} & $0.0845^{* * *}$ & $0.1281^{* * *}$ & 0.0306 \\
\hline & & $(0.0257)$ & $(0.0272)$ & $(0.0443)$ \\
\hline \multirow{2}{*}{ Presión fiscal } & \multirow{2}{*}{$\mathrm{H} 3(+)$} & $0.1558^{* * *}$ & $0.1525^{* *}$ & $0.1787^{* *}$ \\
\hline & & $(0.0528)$ & $(0.0576)$ & $(0.0831)$ \\
\hline \multirow{2}{*}{ Rivalidad política } & \multirow{2}{*}{$\mathrm{H} 4(+)$} & 0.0007 & -0.0016 & 0.0045 \\
\hline & & $(0.0039)$ & $(0.0060)$ & $(0.0043)$ \\
\hline \multirow{2}{*}{ Ideología } & \multirow{2}{*}{$\mathrm{H} 5(?)$} & $0.0630^{* * *}$ & $0.0582^{*}$ & $0.1161^{* * *}$ \\
\hline & & $(0.0147)$ & $(0.0284)$ & $(0.0253)$ \\
\hline \multirow{2}{*}{ Efecto regional } & & $0.0401^{*}$ & -0.0075 & $0.1119^{* * *}$ \\
\hline & & $(0.0213)$ & $(0.0201)$ & $(0.0327)$ \\
\hline \multirow{2}{*}{ Año 2014} & & $0.2728^{* * *}$ & & \\
\hline & & $(0.0391)$ & & \\
\hline \multirow{2}{*}{ Constante } & & $-1.8337^{* * *}$ & $-2.2384^{* * *}$ & $-2.3132^{*}$ \\
\hline & & $(0.5052)$ & $(0.4524)$ & 1.2221) \\
\hline $\mathrm{N}$ & & 200 & 100 & 100 \\
\hline $\mathrm{R}^{2}$ & & 54.1 & 19.4 & 16.2 \\
\hline $\mathrm{F}(7,16)$ & & $138.49^{* * *}$ & & \\
\hline $\begin{array}{l}\text { Breusch-Pagan /Cook- } \\
\text { Weisberg test, p-valor }\end{array}$ & & \multicolumn{3}{|c|}{$\mathrm{X}_{(32)}^{2}=25.44 ; 0.7879$} \\
\hline $\begin{array}{l}\text { Ramsey Reset test, } \\
\text { p-valor }\end{array}$ & & \multicolumn{3}{|c|}{$F_{(3,189)}=0.42 ; 0.7410$} \\
\hline
\end{tabular}

Nota: Figuran entre paréntesis los errores estándares robusto a nivel de clúster por regiones (Comunidades Autónomas).

${ }^{* * *}=$ Significativa para $\mathrm{p}<0.01 ;^{* *}=$ Significativa para $\mathrm{p}<0.05 ;^{*}=$ Significativa para $\mathrm{p}<0.1$

Fuente: Elaboración propia. 
Francisca Tejedo-Romero. Profesora Contratada Doctora de la Universidad de Castilla-La Mancha. Líneas de investigación: transparencia informativa; medición, valoración, gestión y divulgación del capital intelectual; Responsabilidad Social y Buen Gobierno Corporativo. Publicaciones recientes: Joaquim Filipe Ferraz Esteves-Araujo, y Tejedo-Romero, Francisca, "Does Gender Equality Affect Municipal Transparency: The Case of Spain", en Public Performance \& Management Review, núm. 41 vol. 1, Reino Unido: Taylor \& Francis (2018) [disponible en: http://www.tandfonline.com/doi/abs/10.1080/15309576.2 017.1362350?journalCode=mpmr20]; Tejedo-Romero, Francisca, "Información de los recursos intangibles ocultos: ¿memorias de sostenibilidad o informe anual?", en European Research on Management and Business Economics, vol. 22, núm. 2, España: European Academy of Management and Business Economics (2016) [disponible en: https://doi.org/10.1016/j.iedee.2015.06.001]; Tejedo-Romero, Francisca, "Información del conocimiento organizacional a través de los informes anuales publicados en las páginas web de las empresas”, en Revista Española de Documentación Cientifica, vol. 37, núm. 1, España: Centro Nacional de Información y Documentación Científica (2014) [disponible en: http://dx.doi.org/10.3989/redc.2014.1.1068].

Joaquim Filipe Ferraz Esteves Araujo. PhD en Administración Pública por la University de Exeter (Reino Unido). Profesor de la Universidad do Minho (Portugal). Investigador del Centro de Investigación en Ciencia Política de la Universidad do Minho. Director del Programa de Doctorado en Ciencias de la Administración de la Universidad do Minho. Líneas de investigación: gestión pública, transparencia, gobernación. Publicaciones recientes: Joaquim Filipe Ferraz Esteves-Araujo y Tejedo-Romero, Francisca, "Does Gender Equality Affect Municipal Transparency: The Case of Spain”, en Public Performance \& Management Review, núm. 41, vol. 1, Reino Unido: Taylor \& Francis (2018) [disponible en: http://www.tandfonline.com/doi/abs/10.1080/15309576.2017.1362350 ?journalCode=mpmr20]; Pereira, Cleber, Joaquim Filipe Ferraz Esteves-Araujo, y Machado, Maria de Lourdes, "The Brazilian Higher Education Evaluation Model: "SINAES” sui generis?", en International Journal of Educational Development, vol. 61, Reino Unido: W.I. Ozanne and Associates (2018) [disponible en: https://doi.org/10.1016/j.ijedudev.2017.11.007]; Joaquim Filipe-Araujo, y Consciência Silvestre, Hugo, "As Parcerias Público Privadas em Portugal: experiência recente", en Revista de Administração Pública, vol. 48, núm. 3, Brasil: Escola Brasileira de Administracao Publica da Fundacao Getulio Vargas (2014) [disponible en: http://dx.doi.org/10.1590/0034-76121606].

Recepción: 15 de febrero de 2018.

Aprobación: 17 de mayo de 2018. 